\title{
Dissipative Nambu systems and oscillator circuit design
}

\author{
Wolfgang Mathis ${ }^{1 a)}$ and Richard Mathis ${ }^{2}$ \\ ${ }^{1}$ Institute of Theoretical Electrical Engineering, Leibniz Universität Hannover, \\ Appelstrasse 9A, 30167 Hannover, Germany \\ ${ }^{2}$ Department of Physics, University of Göttingen \\ 37077 Göttingen, Friedrich-Hund-Platz 1, Germany \\ a)mathis@tet.uni-hannover.de
}

Received January 5, 2014; Published July 1, 2014

\begin{abstract}
Electronic oscillators have to be described by nonlinear differential equations with a limit cycle where analytical solutions are rarely predictable. Therefore, design concepts of oscillators are not available where a designer can start with a complete set of specifications of the desired oscillator. Only linear aspects of the oscillator design can be solved in a reasonable manner. In this paper we present a mathematical concept that is appropriate to construction nonlinear differential equations with a limit cycle. For this purpose we use a concept from theoretical physics, the so-called Nambu mechanics, and add canonical dissipation. It can be shown that a limit cycle arises under certain conditions. Based on the corresponding system of differential equations a SIMULINK model is constructed that can be the basis of a circuit realization.
\end{abstract}

Key Words: nonlinear circuits, oscillators, dynamical systems, limit cycles, Nambu mechanics, canonical dissipation

\section{Introduction}

Sinusoidal oscillators belonged to the first electronic circuits (e.g. [16]) and many papers as well as monographs are available where various circuit shapes of electronic oscillators [28] with different electronic devices and associated design recipes are presented; see e.g. [9, 10,29] for further details and recent references. However, nonlinear circuit models are needed to understand the functionality of oscillator circuits. Since analytical solutions of nonlinear circuit equations are rarely available the corresponding inverse problems based on the specifications of oscillators cannot be solved [4] and that is why a complete model-based design process for oscillator circuits that includes nonlinear and noise specifications is missing until now. On the other hand, many authors studied mathematical aspects of the electronic oscillators (see e.g. $[1,6,14]$ for further details and references) that can be used for developing a complete model-based design concept in the future.

In this paper we study an approach for the construction of nonlinear differential equations with a limit cycle that can be appropriate for the synthesis of electronic oscillators. For this purpose, we discuss different variants for describing oscillator circuits by nonlinear differential equations which 
admit limit cycles. It turns out that concepts from theoretical mechanics can be very suitable to construct this kind of differential equations.

It is well-known that the describing equations of nonlinear circuits can be formulated as an implicit quasilinear differential system (e.g. [22])

$$
A(x) \dot{x}=f(x)+g(x) u(t)
$$

where $A: \mathbb{R}^{n} \rightarrow \mathbb{R}^{n \times n}, f: \mathbb{R}^{n} \rightarrow \mathbb{R}^{n}, g: \mathbb{R}^{n} \rightarrow \mathbb{R}^{n}$ and $u: \mathbb{R} \rightarrow \mathbb{R}$ with circuit variables $x: \mathbb{R} \rightarrow \mathbb{R}^{n}$. But even if a generalized nodal potential description (e.g. MNA [13]) is used where $A(x)$ and $g(x)$ are constant functions (e.g. [27]) these equations can be solved only in very simple cases. In the following we consider circuit equations of electronic oscillators where no input function $u(t)$ occurs and a limit cycle arises as an asymptotic solution. Furthermore, we restrict us to cases where the circuit variables $x$ are chosen in such a manner that oscillator circuits can be formulated as a family of differential equations $[11,17]$

$$
\dot{x}=f(x, \lambda),
$$

where $f: \mathbb{R}^{n} \rightarrow \mathbb{R}^{n}$ and $\lambda \in \mathbb{R}$ is a certain circuit parameter. From a mathematical point of view these equations describe an electronic oscillator if an Andronov-Hopf bifurcation arises for a certain value $\lambda_{0}$ (e.g. [14]). The sufficient conditions for such a bifurcation are included as assumptions for the theorem of Andronov and Hopf (e.g. [11]). Of course, it is a local approach since we consider the differential equations near the bifurcation point $\lambda_{0}$.

For a global approach we can construct differential equations with a periodic behavior based on the concept of canonical dissipative (CD) systems; see [5] for details and further references. In circuit theory this concept is discussed by $[19,34]$. A necessary condition of the Andronov-Hopf theorem is that the considered family of differential equations is non-hyperbolic in a fix point for some bifurcation point $\lambda_{0}$. A non-hyperbolic fix point is related to a subsystem where the energy is conserved. Since systems with energy conservation are described in physics by the Hamiltonian formalism a close relationship should exist between certain systems with limit cycles and Hamiltonian systems. Accordingly, we can use a Hamiltonian system for constructing the differential equations of a dynamic systems with a limit cycle and add dissipation. Most design concepts of sinusoidal electronic oscillators follow this line that we start with a LC circuit or another resonant subcircuit and compensate the resistive losses. Using some kind of nonlinear control device the amplitude of the circuit is stabilized; see [17] for further details. If the areas of active and passive dissipation in the state space of a circuit are separated by a energy shell of a Hamiltonian system we obtain the concept of CD systems. A comparison of these approaches by simple examples can be found in section 2 .

Unfortunately, the concept of CD systems is too restrictive for electronic circuits since the dimension of the state space of a CD system is even by construction. As mentioned above, the Hamiltonian function is used to construct a CD system with a limit cycle. Since a Hamiltonian function is a first integral of the corresponding Hamiltonian system it is obvious to consider a generalization of Hamiltonian systems with additional first integrals. Some of these integrals are known as Casimir functions; see [15] with respect to electrical systems. Nambu [23] discussed such a concept that is known as Nambu mechanics, see also [21,32]. More recently, some authors studied the properties to some nonlinear dynamic systems with limit cycles within the framework of the Nambu mechanics [3, $26,30]$. A preliminary discussion in the context of electrical oscillators is given by [18].

The paper is organized as follows: In section 2 we will give a comprehensive presentation of CD systems. In section 3 we consider the generalization of Hamilton to Nambu systems and discuss the extension to dissipative Nambu systems. In section 4 we discuss the construction of differential equations with limit cycles using the concept of dissipative Nambu mechanics and present in section 5 a associated electronic systems. In section 6 we end up with some conclusions.

\section{Canonical dissipative systems}

At first, we define the concept of Hamiltonian systems and their canonical dissipative augmentation. For this purpose we consider systems with a configuration space of $n$ degrees of freedom 
$i=1, \ldots, n$ and the coordinates $\left(q_{1} \ldots, q_{n}\right)$ as well as a $2 n$-dimensional state space with the coordinates $\left(q_{1} \ldots, q_{n} ; p_{1}, \ldots, p_{n}\right)$. Then, let be $H\left(q_{1} \ldots, q_{n} ; p_{1}, \ldots, p_{n}\right)$ a smooth real-valued Hamiltonian function over state space $\mathbb{R}^{2 n}$. The equations of motion are defined by [2]

$$
\frac{d q_{i}}{d t}=\frac{\partial H}{\partial p_{i}}, \quad \frac{d p_{i}}{d t}=-\frac{\partial H}{\partial q_{i}},
$$

for $i=1, \ldots, n$. From these equations it can be shown very easily that $d H / d t=0$ such that the Hamiltonian function $H$ is a first integral of the corresponding dynamical system. $H$ is interpretable as the energy of the system. Therefore, the state space can be decomposed into energy surfaces that are defined by the zero sets $H\left(q_{1} \ldots, q_{n} ; p_{1}, \ldots, p_{n}\right)=E=$ const. If the initial point of a solution $\left\{q_{i}=q_{i}(t) ; p_{i}=p_{i}(t) \mid i=1, \ldots, n\right\}$ of the equations of motion (3) is contained in a certain energy surface the whole trajectory is included in this energy surface.

It is well-known that a simple example of a Hamiltonian system in electrical circuits is the LC circuit (see [33] and [15]). The circuit equations can be formulate for the charge $q \equiv q_{1}$ of the capacitor and the flux $\phi \equiv p_{1}$ of the inductor in the following manner

$$
\begin{aligned}
\dot{q} & =\frac{\phi}{L}, \\
\dot{\phi} & =-\frac{q}{C}
\end{aligned}
$$

with the Hamiltonian function

$$
H(q, \phi)=\frac{q^{2}}{2 C}+\frac{\phi^{2}}{2 L} .
$$

An alternative formulation of the Hamiltonian equations of motion can be presented if the so-called Poisson brackets are defined

$$
\{F, G\}:=\sum_{i=1}^{n} \frac{\partial F}{\partial q_{i}} \frac{\partial G}{\partial p_{i}}-\frac{\partial F}{\partial p_{i}} \frac{\partial G}{\partial q_{i}}
$$

for differentiable real-valued functions $G(q, p), F(q, p)$ with $(q, p):=\left\{p_{i}, q_{i} \mid i=1, \ldots, n\right\}$.

Then the Hamiltonian equations of motion can be rewritten as

$$
\frac{d q_{i}}{d t}=\left\{q_{i}, H\right\}=\frac{\partial H}{\partial p_{i}}, \quad \frac{d q_{i}}{d t}=\left\{p_{i}, H\right\}=-\frac{\partial H}{\partial q_{i}}
$$

for $i=1, \ldots, n$.

Now, we define a first kind of dissipative systems based on a Hamiltonian system by adding dissipation using a real-valued function $g(H)$. The equation of motion is formulated in the following manner [5]

$$
\begin{aligned}
\frac{d q_{i}}{d t} & =\frac{\partial H}{\partial p_{i}}, \\
\frac{d p_{i}}{d t} & =-\frac{\partial H}{\partial q_{i}}-g(H) \frac{\partial H}{\partial p_{i}}
\end{aligned}
$$

for $i=1, \ldots, n$. Ebeling et al. [5] refer to these equations as canonical dissipative (CD) system [19]. Furthermore, it is possible to augment also the other equations with a dissipative term.

In general, the energy is not conserved for CD systems (8), i. e. we have

$$
\frac{d H}{d t}=-g(H) \sum_{i=1}^{n}\left(\frac{\partial H}{\partial p_{i}}\right)^{2} \neq 0
$$

There are several possibilities to choose the dissipative function $g(H)$ such that the energy $H$ is conserved on a subset of the state space. In the simplest case we can choose a constant dissipation function $g(x)=\gamma_{0}>0$ for all admitted $x \in \mathbb{R}$. It results from (11) that the energy function $H$ decreases to zero and therefore the solutions $(q(t), p(t)):=\left\{q_{i}=q_{i}(t) ; p_{i}=p_{i}(t) \mid i=1, \ldots, n\right\}$ converge to zero, too. 


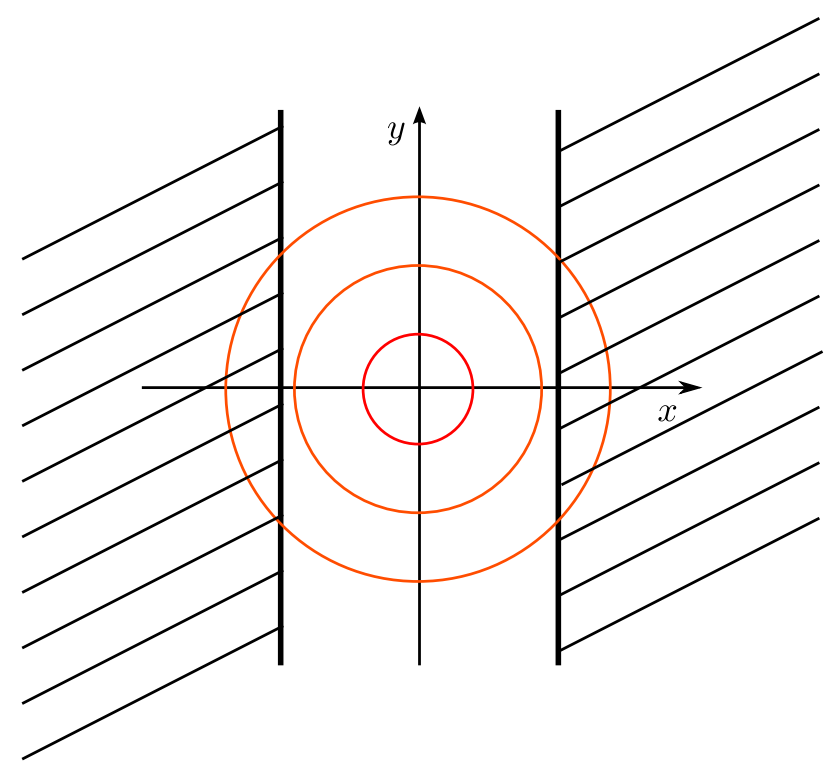

Fig. 1. State space of the van der Pol equation with damping areas.

A more interesting case is obtained if we choose an increasing dissipation function $g(H)$ which changes its sign for $H(q(t), p(t))=E^{*}$ where $E^{*}$ is a certain energy value. Then, for all states $(q(t), p(t))$ energy will be dissipated from the system as long as the inequality $H(q(t), p(t))>E^{*}$ is satisfied whereas for $H(q(t), p(t))<E^{*}$ energy is pumped into the system. For $H(q(t), p(t))=E^{*}$ the dissipation function $g(H)$ vanishes and the CD system reduces to a Hamiltonian system. The solutions $(q(t), p(t))$ of the reduced system are contained within the boundary defined by the condition $H(q, p)=E^{*}$ in the state space which separates the areas of positive damping - dissipation - and negative damping - energy supply. The theorem of uniqueness for the equations of motion [12] does not allow a crossing of solutions such that all solutions outside and inside the boundary set converge to it, respectively. A simple dissipation function $g(H)$ with the properties prescribed above is affine in $H$, i. e.

$$
g(H)=C\left(H-E^{*}\right)
$$

where $C$ is a real constant.

In order to illustrate different possibilities for introducing a dissipation term into a Hamiltonian system we consider a 2-dimensional state space with the Hamiltonian function

$$
H(x, y)=\frac{1}{2}\left(x^{2}+y^{2}\right) .
$$

Obviously, $H(x, y)$ is closely related to the Hamiltonian function (6) of the LC circuit (without damping). By means of $H(x, y)$ the equations of motion can be derived by

$$
\begin{aligned}
\dot{x} & =\frac{\partial H}{\partial y}=y, \\
\dot{y} & =-\frac{\partial H}{\partial x}=-x
\end{aligned}
$$

that are equivalent to the differential equation

$$
\ddot{x}+x=0
$$

with sinusoidal solutions $x(t)=A \sin (\omega t+\varphi)$.

The energy surfaces $H(x, y)-E=\left(x^{2}+y^{2}\right) / 2-E=0$ for positive energies values $E>0$ in the state space $\mathbb{R}^{2}$ correspond to a $E$-parametrized family of circles with the radius $\sqrt{E}$ covering the entire 2-dimensional state space $\mathbb{R}^{2}$.

Now, we add energy dissipation to the system. In the simplest case we add the term $-\gamma y$ with a constant function $g(H)=\gamma>0$ to the Eq. (15) and $+\gamma \dot{x}$ to Eq. (16), respectively, and obtain a 


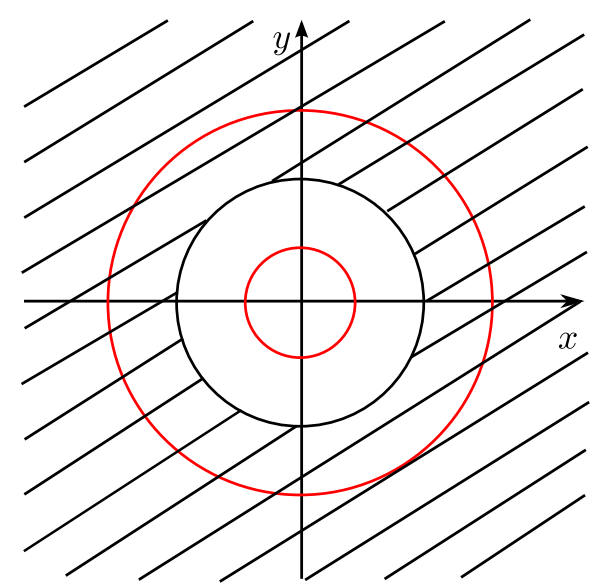

Fig. 2. State space of the Rayleigh-van der Pol equation with damping areas.

linear harmonic oscillator with damping. If the term $\left(x^{2}-1\right) y$ is added to Eq. $(15)$ or $+\left(x^{2}-1\right) \dot{x}$ in Eq. (16) we obtain the van der Pol equation

$$
\ddot{x}+\epsilon\left(x^{2}-1\right) \dot{x}+x=0 .
$$

Note, that it is not a CD system. Obviously, negative dissipation arises for all $\left|x^{2}-1\right|<1$ whereas positive dissipation arises outside this vertical strip, see Fig. 1. In this case we obtain a limit cycle if the supplied and dissipated energy are in a dynamic equilibrium. Obviously, the limit cycle cannot be a circle.

If the dissipative term $\left(x^{2}+y^{2}-1\right) y$ is added to Eq. (15) or $+\left(x^{2}-1\right) \dot{x}$ in Eq. (16) the boundary of the supplied and dissipated energy corresponds to a circle and a energy shell of the Hamiltonian system where periodic exchanges of energy do not occur. In Fig. 2 the area of positive damping is dashed whereas in the inner cycle there is negative damping. The extended differential equation of Eq. (16)

$$
\ddot{x}+\epsilon\left(x^{2}+\dot{x}^{2}-1\right) \dot{x}+x=0 .
$$

is called Rayleigh-van der Pol equation (e.g. [17,24]). Since the dissipation term vanishes on the circle the remaining differential equation is equivalent to a Hamiltonian system with the Hamiltonian function (13). This is the main idea the construction of limit cycles with a CD systems. It is interesting to note that a stochastic behavior can be incorporated into a CD system by means of an additive white noise term; see for further details [5,34], and [19].

From a physical point of view CD systems cannot be realized in nature since the dynamics of the system for states in the boundary area between positive and negative damping is completely decoupled from the rest of the world. Of course, this is not possible in real systems. However, some physical systems can be modeled by a CD system as a good approximation.

For example, crystal oscillators consist of resonators with a very high Q factor and therefore very small portions of energy have to be pumped into the circuit within one cycle; see [8]. For this reason, after the transient a crystal oscillator circuit behaves itself in a very good approximation like a Hamiltonian system and entirely it can be described as a CD system. The same arguments can be used if we consider other sinusoidal oscillators which include resonators with a high Q factor. Some realization concepts of CD systems with circuit and system models are discussed in [19]. In Fig. 3 a circuit realization of the Rayleigh-van der Pol equation (18) is shown.

Although the van der Pol oscillator (17) and its differential equation cannot be a CD system we obtain an approximative CD system if an averaging perturbation method is applied; see e.g. [25]. Reformulating the van der Pol equation in polar coordinates the following perturbation equations are derived

$$
\begin{aligned}
\frac{d R}{d t} & \approx \frac{1}{2} R\left(1-\frac{R^{2}}{4}-\cos 2 \theta+\frac{R^{2}}{4} \cos 4 \theta\right), \\
\frac{d \theta}{d t} & \approx-1+\frac{1}{2} \sin 2 \theta-\frac{1}{4} \sin 2 \theta-\frac{1}{8} \sin 4 \theta .
\end{aligned}
$$




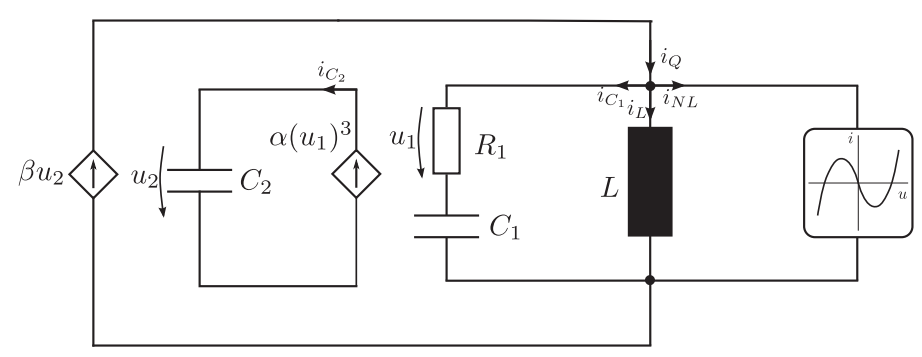

Fig. 3. A circuit realization of the Rayleigh-van der Pol equation.

After an averaging process over one period we obtain the following differential equations

$$
\begin{aligned}
\frac{d \tilde{R}}{d t} & \approx \frac{1}{2} R\left(1-\frac{R^{2}}{4}-\int_{0}^{2 \pi} \cos 2 \theta d \theta+\int_{0}^{2 \pi} \frac{R^{2}}{4} \cos 4 \theta d \theta\right)=\frac{1}{2} \tilde{R}\left(1-\frac{\tilde{R}^{2}}{4}\right), \\
\frac{d \tilde{\theta}}{d t} & \approx-1+\int_{0}^{2 \pi}\left(\frac{1}{2} \sin 2 \theta-\frac{1}{4} \sin 2 \theta-\frac{1}{8} \sin 4 \theta\right) d \theta=-1
\end{aligned}
$$

which approximates the van der Pol equation. In original coordinates the approximative differential equations corresponds to the Rayleigh-van der Pol equation with $H(q, p)=\left(q^{2}+p^{2}\right) / 2$ and $g(H)=$ $H-4$ and therefore a CD system. In general, 2-dimensional nonlinear dynamical systems with a limit cycle can be approximated by a CD system at least in a first order approximation process.

In higher dimensional state spaces of a CD systems the energy surfaces are hypersurfaces such that a canonical dissipative damping term in the equations of motion leads in asymptotic stable cases to a dynamics on a hypersurface. Only in 2-dimensional state spaces these hypersurfaces are 1-dimensional submanifolds. Therefore, the concept of CD systems is suitable for the construction of sinusoidal electronic oscillators where a (1-dimensional) limit cycle is needed only if the state space is 2-dimensional. However, if we need a more complex periodic behavior - e.g. trajectories on a torus in the 3-dimensional case - the concept of CD systems can be used.

\section{Dissipative Nambu systems}

In 1973, Yoichiro Nambu [23] developed a generalization of the classical Hamiltonian mechanics where more than one first integral is used to derive the equations of motions. These first integrals are referred to as Nambu-Hamiltonian functions. In our paper we use the concept of Poisson-Nambu brackets for representing Nambu's equations of motion that generalizes the Poisson brackets.

In order to formulate the equations of motion of a Nambu system with $n$ degrees of freedom we need $n-1$ Nambu-Hamiltonian functions. In the 2-dimensional case we have only one Hamiltonian function and the Nambu system reduces to an ordinary Hamiltonian system.

At first, we define the Poisson-Nambu bracket of a triple of Nambu-Hamiltonian functions $f_{1}, f_{2}, f_{3}$ in dependency of the coordinates $x, y, z$

$$
\left\{f_{1}, f_{2}, f_{3}\right\}=\frac{\partial\left(f_{1}, f_{2}, f_{3}\right)}{\partial(x, y, z)} .
$$

Note, that the right-hand side corresponds the Jacobian matrix of the vector field $\vec{f}=\left(f_{1}, f_{2}, f_{2}\right)$ : $\mathbb{R}^{3} \rightarrow \mathbb{R}^{3}$. The generalized Nambu-Hamiltonian equations of motion or Nambu system with two Nambu-Hamiltonian functions $H_{1}$ and $H_{2}$ can be formulated as follows

$$
\frac{d \vec{f}}{d t}=\left\{H_{1}, H_{2}, \vec{f}\right\}
$$

In the 3-dimensional case the right-hand side can be reformulated by means of gradients of the Nambu-Hamiltonian functions

$$
\frac{d \vec{f}}{d t}=\nabla H_{1} \times \nabla H_{2}
$$


It is easy to show that Nambu dynamics on the state space is divergence free or solenoidal, i.e. the divergence of the Nambu vector field vanishes: $\operatorname{div}\left(\nabla H_{1} \times \nabla H_{2}\right)=0$. Furthermore, Nambu systems conserve the volume in the state space like in the case of Hamiltonian systems due to the Liouville theorem.

As an example we consider the following differential equations

$$
\begin{aligned}
& \frac{d x}{d t}=\sigma y, \\
& \frac{d y}{d t}=x(r-z), \\
& \frac{d z}{d t}=x y
\end{aligned}
$$

which represent the non-dissipative part of the Lorenz equations [3, 26]. Using the Nambu-Hamiltonian functions

$$
\begin{aligned}
& H_{1}=\frac{1}{2}\left(y^{2}-(z-r)^{2}\right), \\
& H_{2}=\sigma z-\frac{x^{2}}{2}
\end{aligned}
$$

the differential equations (24) can be reconstructed by calculating the Nambu vector field $\nabla H_{1} \times \nabla H_{2}$ and inserts it into Eq. (23). Therefore, we refer to these equations as Nambu-Lorenz system.

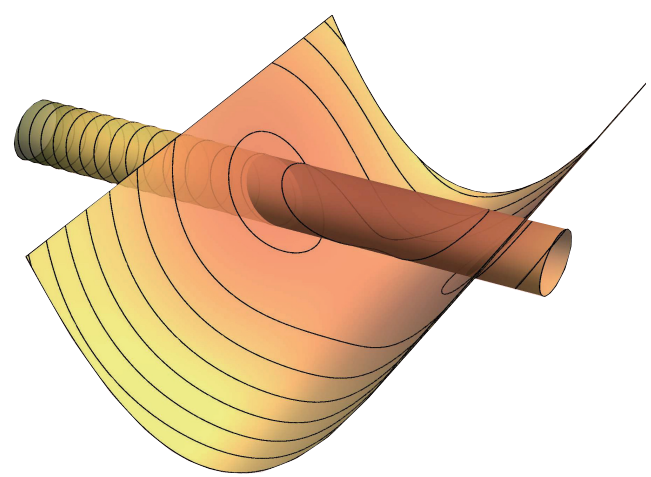

Fig. 4. Intersection of the Nambu-Hamiltonian functions.

A geometrical interpretation of the Nambu-Hamiltonian functions $H_{1}$ and $H_{2}$ can be presented if we consider the zero sets of these functions and obtain a cylindric and a parabolic surface, see Fig. 4. Both constrains have to be fulfilled such that the solution trajectories of the Nambu-Lorenz system are included in the intersection of the two surfaces, asymptotically. Obviously, the 3-dimensional case of Nambu-Poisson brackets can be easily generalized to $n$-dimensional case where we have $n$ NambuHamiltonian functions $H=\left(H_{k}\right): \mathbb{R}^{n} \rightarrow \mathbb{R}(k=1, \ldots, n-1)$. Using this definition, we obtain the equations of motion for the $n$-dimensional Nambu-system $(i=1, \ldots, n)$

$$
\frac{d f_{i}}{d t}=\frac{\partial\left(f_{i}, H_{1}, \ldots H_{n-1}\right)}{\partial\left(f_{1}, \ldots, f_{n}\right)} .
$$

If the Nambu system is $n$-dimensional we have $n-1 \mathrm{Nambu-Hamiltonian} \mathrm{functions} H_{i}$ where the trajectories of the system are within the intersection of the corresponding surfaces of $H_{i}$ if the intersections are transversal; see [7].

Now, we are able to use the concept of CD systems to incorporate dissipation into Nambu systems. For illustration we consider a linear damping term in 3-dimensional state space where we have two Nambu-Hamiltonian functions $H_{1}$ and $H_{2}$. Following Frank in his seminal paper [7] we use a canonical dissipative damping term in the form

$$
D g\left(H_{1}, H_{2}\right):=\gamma\left(\nabla H_{1}\left(H_{1}-E_{1}\right)+\nabla H_{2}\left(H_{2}-E_{2}\right)\right) .
$$


If this $D g$ is added to the equations of motion (23) for the Nambu-Hamiltonian functions $H_{1}, H_{2}$ we obtain the CD Nambu equations

$$
\dot{\vec{f}}=\nabla H_{1} \times \nabla H_{2}-D g .
$$

In the next two sections we study a Nambu system with canonical dissipation and its electronic realization in more details. It should be mentioned that also other authors studied Nambu systems with dissipation; see e.g. [3]. However, in contrast to systems with canonical dissipation the asymptotic dynamic behavior is more complex and and the trajectories cannot be evaluated by in a geometric manner.

\section{Modified Lorenz system as CD Nambu system}

In order to illustrate the asymptotic behavior of a CD Nambu system we consider the well-known Lorenz equations and with its state space $\mathbb{R}^{3}$

$$
\begin{aligned}
& \frac{d x}{d t}=\sigma y-\sigma x, \\
& \frac{d y}{d t}=x(r-z)-y, \\
& \frac{d z}{d t}=x z-b z
\end{aligned}
$$

with $\sigma=10, r=28$, and $b=8 / 3$. In addition to Eq. (24) linear damping parts are included in each Eq. (30)-(32).

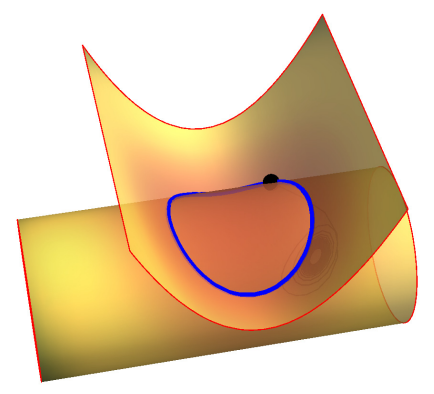

Fig. 5. Intersection of Nambu-Hamiltonian Surfaces at time $t=0$.

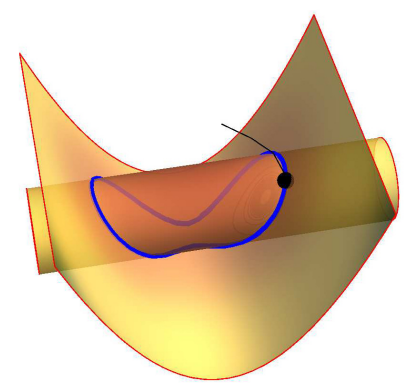

Fig. 6. Intersection of Nambu-Hamiltonian Surfaces at time $t=10$.

It is mentioned above, that the non-dissipative part of these equations (24) can be reformulated as a Nambu system and the Nambu-Hamiltonian functions can be represented by a cylindric and a parabolic surface. The Lorenz equations with the constant values for $\sigma, r$ and $b$ allow chaotic behavior (e.g. [31]) that can be understood in more details if the Nambu-Hamiltonian functions and their geometrical interpretation are used; see [3].

For the non-dissipative Nambu-Lorenz equations (24) all trajectories are included in the intersection of the two surfaces that is not changing in dependency of time. Now, canonical dissipation is added to the Eq. (24) in the sense of Eq. (29) and (28) using the Nambu-Hamiltonian functions (25) 


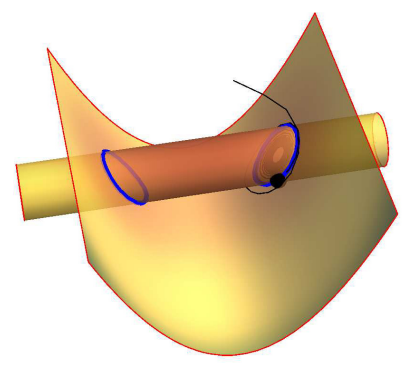

Fig. 7. Intersection of Nambu-Hamiltonian Surfaces at time $t=70$.

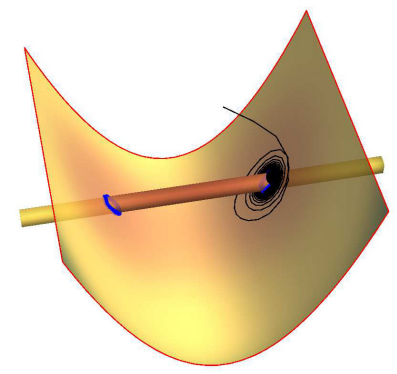

Fig. 8. Intersection of Nambu-Hamiltonian Surfaces at time $t=200$.

and (26). We denote these differential equations as CD Lorenz-Nambu system. Furthermore, the parabolic surface is unchanged for all times $t>0$; see Figs. 5-8 (initial conditions: $x_{0}=8, y_{0}=10$, $z_{0}=20$ ). But the cylindric surface that it is specified by its radius is changing with $t$. From a physical point of view that radius is proportional to the energy of the system. A trajectory starts from an arbitrary initial point in the state space and converges into the intersection of the two surfaces. The dynamics of the intersection depends in an essential manner on the initial values of the trajectory. If the initial values are chosen such that the energy of the system is too small and the trajectory starts inside the predicted limit cycle and energy has to be pumped into the system. Otherwise, the energy of the system has to be reduced. Finally, the trajectory converges into the desired intersection of the two surfaces and is stable. The Figs. 5-8 the dynamics of the intersection of the surfaces in dependency of the scaled time $t$ are shown. Although the intersection with respect to the initial conditions is simply connected it becomes decomposed into two components during the dynamical evolution; see Fig. 6 and Fig. 7. Finally, the dynamics and the trajectory converge to the right component; see Fig. 8 that corresponds to the intersection in Fig. 4.

Finally, we show that the canonical damping term vanishes, asymptotically. In Fig. 9 the behavior of the damping term of the $y$ equation is presented. In dependency of the scaled time $t$ the amplitude of this damping term converges to zero. In the same manner the damping terms of the other equations decrease to zero.

\section{Aspects of electronic realization of the CD Lorenz-Nambu system}

In the last section the Nambu-Lorenz system was augmented with canonical dissipation and we obtained a CD Nambu-Lorenz system with a limit cycle. Now, we discuss some aspects of an electronic realization of this system. As a first step, a SIMULINK model for the CD Nambu-Lorenz system was constructed where only integrators and multipliers are used; see Fig. 10. Therefore, an electronic realization can be constructed by standard electronic devices. A detailed circuit realization and its properties will be a subject of a forthcoming paper.

In Fig. 11 the asymptotic behavior the system is shown. Obviously, the state variables converge to a harmonic steady state that can be identified with the desired limit cycle.

In Fig. 12 the solution $(x(t), y(t), z(t))$ of the CD Nambu-Lorenz starts at an arbitrary initial value and we consider the projection to the $x-y$ coordinate plane. The behavior of the trajectory 


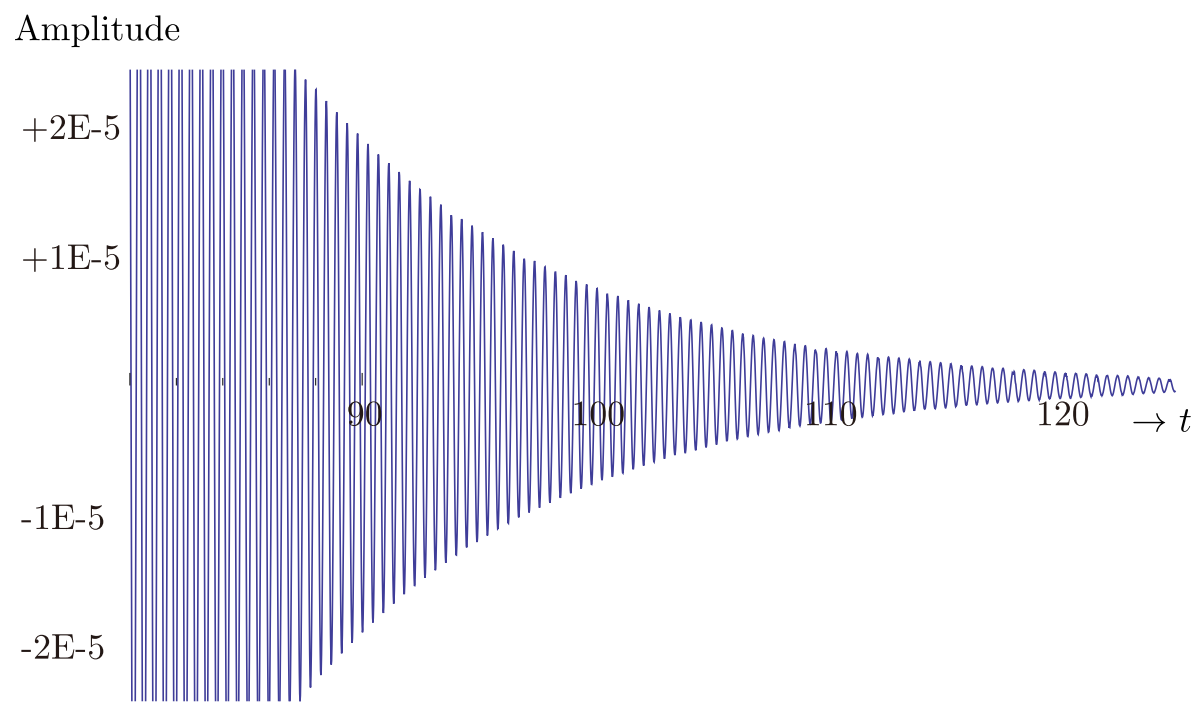

Fig. 9. Canonical Damping Term of the $y$-Equation in the Dependency of $t$.

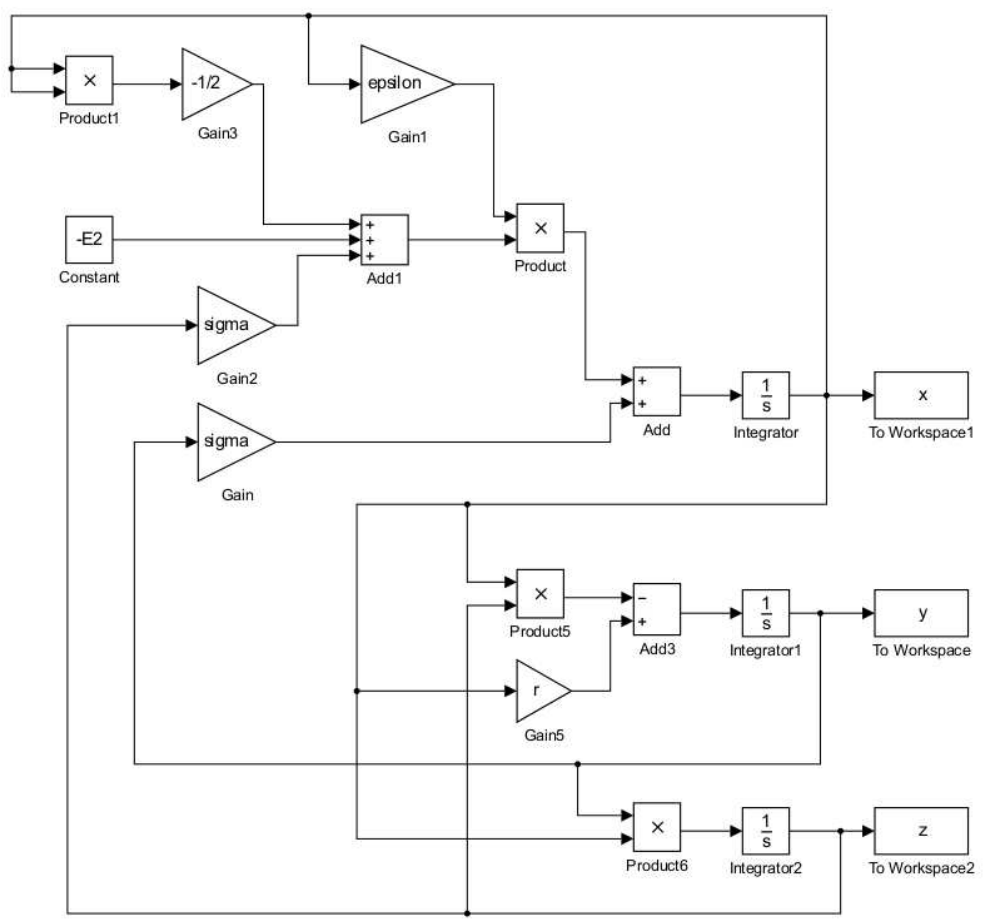

Fig. 10. SIMULINK Realization of the CD Lorenz-Nambu System.

is understandable in geometrical manner if we compare it with the Figs. 5-8 in section 4. But in contrast to Fig. 8 the trajectory converges to the part of the intersection on the left hand side. In Fig. 13 the state trajectory is projected the $z-x$ coordinate plane. We observe that the trajectory is attractive by the surface of the Nambu-Hamilton function $H_{2}$. Finally, in Fig. 14 the state trajectory is projected on the $z-y$ coordinate plane. Both projections can be interpreted geometrically by means of our discussions in section 4 .

\section{Conclusions}

In this paper we discussed some mathematical aspects of the problem of oscillator synthesis where a oscillatory behavior in the state space is prescribed and an electronic circuit can be constructed at the end of the process. Since oscillator circuits have to be described by nonlinear differential equations 


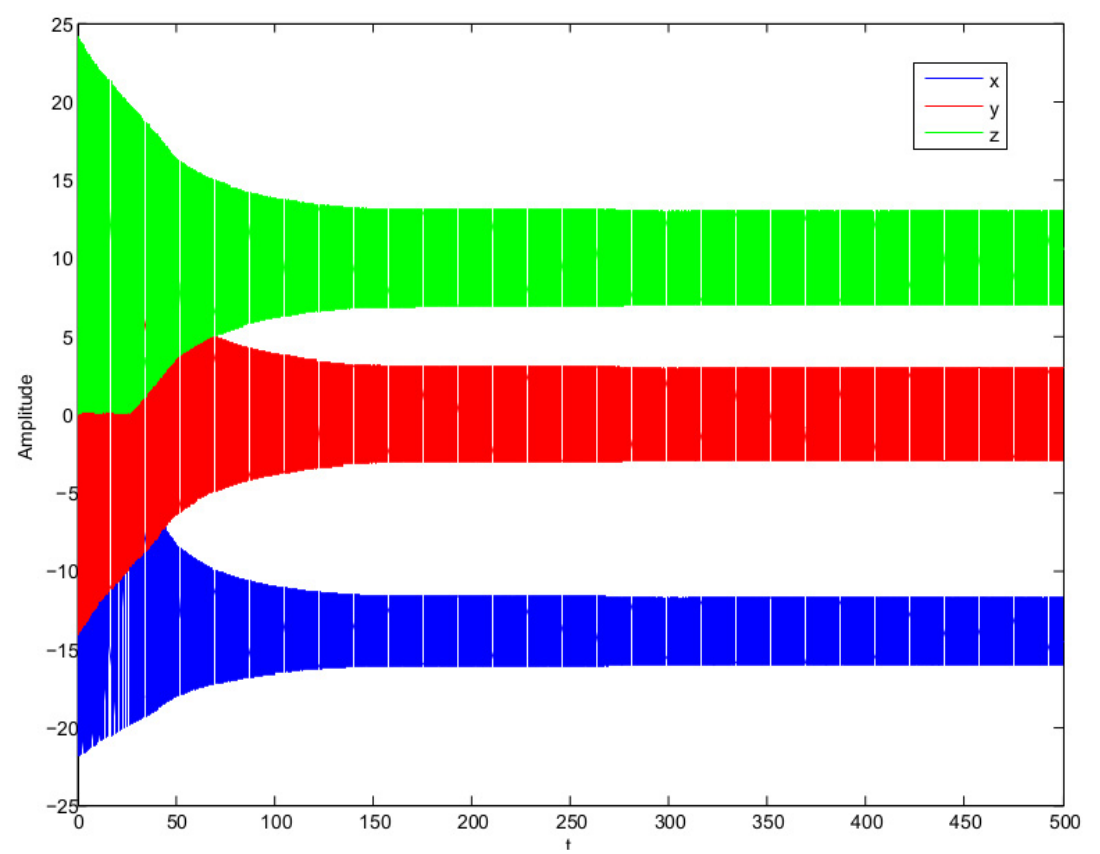

Fig. 11. Asymptotic Behavior of SIMULINK Realization of CD Lorenz System.

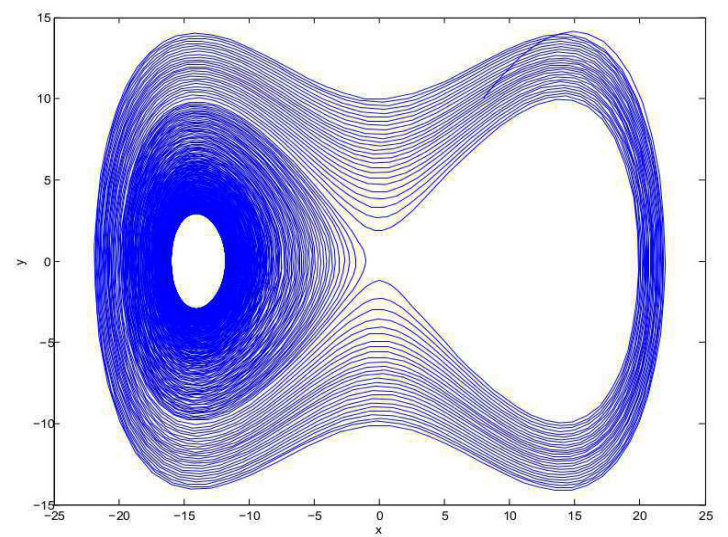

Fig. 12. Projection of the State Trajectory in the $y-x$ Coordinate Plane.

the problem of synthesis is unsolved until now. In order to develop a concept of synthesis for a special class of oscillators we studied the so-called the Nambu mechanics and add canonical dissipation. We were able to show that differential equations with a limit cycle can be constructed where the limit cycle can be prescribed as the intersection of two surfaces in the 2 -dimensional case. In $n$-dimensional cases we have to intersect $n-1$ surfaces. Therefore, a geometrical interpretation of this approach is available. Based on the constructed differential equations a SIMULINK model was developed that can be a starting point of a synthesis of an oscillator circuit. The concept was illustrated by means of differential equations that are related to the well-known Lorenz equations. However, our approach can be applied to other differential equations that are representable as a Nambu system.

\section{Acknowledgments}

We thank M. Sc. Daniel Stahl for some calculations with MATHEMATICA and valuable discussions and we thank Dipl.-Ing. Marco Reit and Dipl.-Ing. Michael Popp for their help with respect to the development of the MATLAB/SIMULINK model as well as discussion about the electronic realization of CD and Nambu systems. Furthermore, we thank Dipl.-Phys. Dipl.-Ing. Florian Richter and Dipl.Ing. Tina Thiessen for their work on CD systems. Finally, we are indebted to Prof. Dr. Rainer 


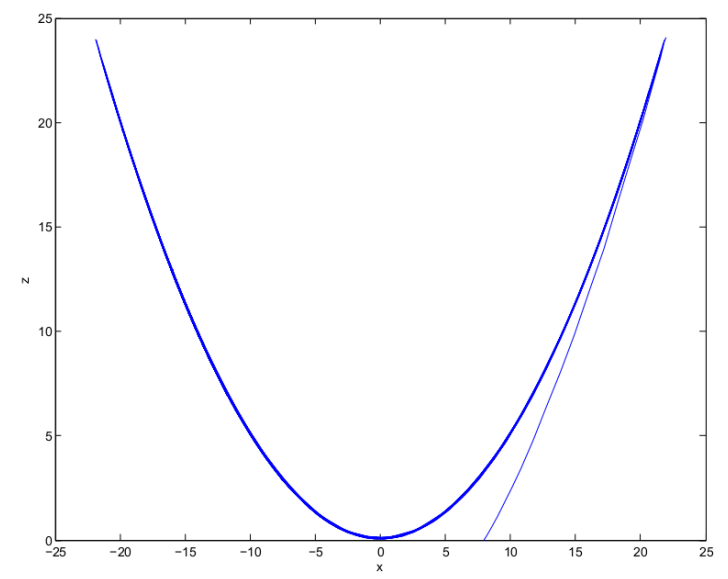

Fig. 13. Projection of the State Trajectory in the $z-x$ Coordinate Plane.

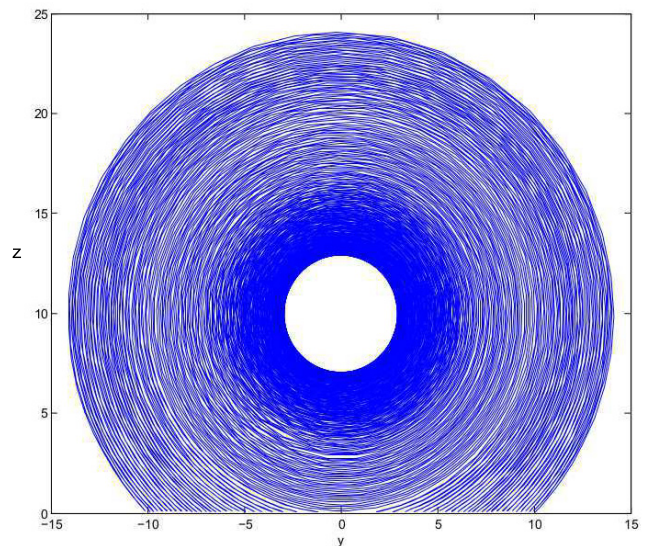

Fig. 14. Projection of the State Trajectory in the $z-y$ Coordinate Plane.

Klages (Queen Mary University London, UK) for many discussions and especially for clarifying the relationship of Nosé-Hoover thermostats and CD systems.

\section{References}

[1] A.A. Andronov, A. Witt, and S. Chaikin, Theory of Oscillators (Reprint), Dover Publication Inc., New York, 1966 (first publication 1937).

[2] V.I. Arnol'd, Mathematical Methods of Classical Mechanics (2. Ed.), Springer-Verlag, BerlinHeidelberg-New York 1989.

[3] M. Axenides and E. Floratos, "Scaling properties of the Lorenz system and dissipative Nambu mechanics," arXiv:1205.3462v2 [nlin.CD], 19 June 2012.

[4] V.L. Chechurin, N.V. Korovkin, and M. Hayakawa, Inverse Problems in Electrical Circuits and Electromagnetic Field Theory, Springer-Verlag, Berlin - New York, 2007.

[5] W. Ebeling and I.M. Sokolov, Statistical Thermodynamics and Stochastic Theory of Nonequilibrium Systems, World Scientific Publ. C. Pte. Ltd., 2005.

[6] H. Fathabadi and S.K. Nikravesh, "A Theoretical Method for Design and Realization of Fixed Amplitude Sinusoidal Oscillators," Analog Integrated Circuits and Signal Processing, vol. 39, pp. 123-130, 2004.

[7] T.D. Frank, "Active systems with Nambu dynamics: with applications to rod wielding for haptic length perception and self-propagating systems on two-spheres," Eur. Phys. J. B, vol. 74, pp. 195-203, 2013.

[8] M.E. Frerking, Crystal Oscillator Design and Temperature Compensation, van Nostrand Reinhold Comp., New York 1978.

[9] G. Gonzalez, Foundations of Oscillator Circuit Design, Artech House, Inc., 2007. 
[10] A. Grebennikov, RF and Microwave Transistor Oscillator Design, John Wiley \& Sons; New York 2007.

[11] J. Guckenheimer and P. Holmes, Nonlinear Oscillations, Dynamical Systems, and Bifurcations of Vector Fields, Springer-Verlag, 1983.

[12] D.W. Jordan and P. Smith, Nonlinear Ordinary Differential Equations, 4. Edition, Oxford University Press, 2007.

[13] C.W. Ho, A.E. Ruehli, and P.A. Brennan, "The modified nodal approach to network analysis," IEEE Trans. Circuits Syst., vol. CAS-22, pp. 504-509.

[14] G.M. Maggio, O. De Feo, and M.P. Kennedy, "A general method to predict the amplitude of oscillation in nearly-sinusoidal oscillators," IEEE Trans. Circuits and Systems I, vol. CAS-I 51, no. 8, pp. 1586-95, 2004.

[15] B.M. Maschke, A.J. van der Schaft, and P.C. Breedveld, "An intrinsic hamiltonian formulation of the Dynamics of LC-Circuits," IEEE Trans. Circuits and Systems I, vol. CAS-I 42, no. 2, pp. 73-83, 1995.

[16] W. Mathis and J. Bremer, "Modelling and design concepts for electronic oscillators and its synchronization," The Open Cybernetics and Systemics Journal, vol. 3, pp. 47-60, 2009.

[17] W. Mathis and P. Russer, "Oscillator design," in: In: K. Chang, Ed., Encyclopedia of RF and Microwave Engineering, vol. 4, pp. 3563-3589, New York: John Wiley, 2005.

[18] W. Mathis, D. Stahl, and R. Mathis, "Oscillator synthesis based on Nambu mechanics with canonical dissipative damping," 21st European Conference on Circuit Theory and Design (ECCTD), September 18-12, Dresden, Germany.

[19] W. Mathis, F. Richter, and R. Mathis, "Stochastic behavior of dissipative hamiltonian systems with limit cycles," Proc. MATHMOD 2012, Vienna, Austria, February 15-17, 2012.

[20] S. Mongkolsakulvong, P. Chaikhan, and T.D. Frank, "Oscillatory nonequilibrium Nambu systems: the canonical-dissipative Yamaleev oscillator," Eur. Phys. J. B, pp. 85-90, March 2012.

[21] N. Mukunda and E.C.G. Sudarshan, "Relation between Nambu and Hamiltonian Mechanics," Phys. Rev. D, vol. 13, no. 10, pp. 2403-2412, 1976.

[22] M.C. Munoz-Lecanda and N. Roman-Roy, "Implicit quasilinear differential systems: a geometrical approach," Electronic Journal of Differential Equations, vol. 1999, no. 10, pp. 1-33, 1999.

[23] Y. Nambu, "Generalized hamiltonian dynamics," Phys. Rev. D, vol. 7, pp.2403-2412, 1973.

[24] A. Nathan, "The Rayleigh-van der Pol harmonic oscillator," Int. J. Electronics, vol. 43, no. 6, pp. 609-614, 1977.

[25] A.H. Nayfeh, Introduction to Perturbation Techniques, John Wiley \& Sons, New York 1993.

[26] P. Névir and R. Blender, "Hamiltonian and Nambu representation of the Non-Dissipative Lorenz Equations," Beitr. Phys. Atmosph., vol. 67, no. 2, pp. 133-140, 1994.

[27] R.W. Newcomb, "The semistate description of Nonlinear time-variable circuits," IEEE Trans. Circ. Syst., vol. CAS-28, no. 1, pp. 62-71, 1981.

[28] T.H. O'Dell, Electronic Circuit Design: Art and Practice, Cambridge University Press, 1988.

[29] U.L. Rohde, A.K. Poddar, and G. Bck, The Design of Modern Microwave Oscillators for Wireless Applications: Theory and Optimization, John Wiley \& Sons, New York, 2005.

[30] Z. Roupas, "Phase space geometry and chaotic attractors in dissipative Nambu mechanics," arXiv:1110.0766v3 [nlin.CD], 25 April 2012.

[31] C. Sparrow, The Lorenz Equations: Bifurcations, Chaos, and Strange Attractors, SpringerVerlag, Berlin - New York, 1982.

[32] W.-H. Steeb and N. Euler, "A note on Nambu mechanics," Il Nuovo Cimento, vol. 106 B, no. 3, pp. 263-272, 1991.

[33] T.E. Stern, Theory of Nonlinear Networks and Systems: An Introduction, Reading, MA: Addison-Wesley, 1965.

[34] T. Thiessen and W. Mathis, "On noise analysis of oscillators based on statistical mechanics," Intern. Journ. of Electronics and Telecommunications, vol. 56, pp. 357-366, 2010. 